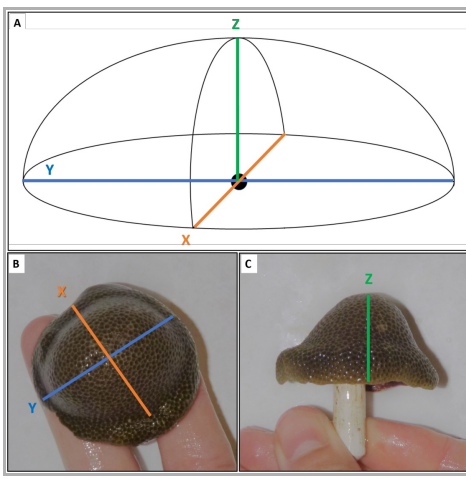

JAN 19, 2021

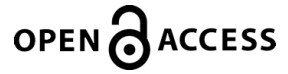

DOI:

dx.doi.org/10.17504/protocol s.io.bpxcmpiw

Protocol Citation: Rowan Mclachlan, Andrea G Grottoli 2021. Geometric Method for Estimating Coral Surface Area Using Image Analysis.

protocols.io

https://dx.doi.org/10.17504/p rotocols.io.bpxcmpiw

License: This is an open access protocol distributed under the terms of the Creative Commons Attribution License, which permits unrestricted use, distribution, and reproduction in any medium, provided the original author and source are credited

Protocol status: Working We use this protocol and it's working

Created: Nov 20, 2020

Last Modified: Jan 19, 2021

PROTOCOL integer ID: 44740

\section{(3) Geometric Method for Estimating Coral Surface Area Using Image Analysis}

In 1 collection

\author{
Rowan Mclachlan ${ }^{1}, \quad$ Andrea G Grottoli ${ }^{1}$ \\ ${ }^{1}$ The Ohio State University
}

Coral Bleaching RCN protocols

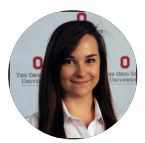

Rowan Mclachlan

The Ohio State University

\section{ABSTRACT}

This protocol outlines a non-destructive geometric method for estimating the surface area of Scleractinian coral samples with relatively simple morphologies (e.g., not densely branching). The geometric method was one of the earliest used for estimating the surface area of marine organisms (Odum et al. 1958). The basic principle of this method involves selecting geometric shapes or forms which closely resemble the morphology of a coral fragment (e.g., cylinders, cones, pyramids, hemispheres etc.), measuring the dimensional parameters of the coral, and applying the area equation for a given geometric shape to obtain the surface area estimate for the coral. This method has been commonly used in coral research previously (Szmant-Froelich 1985; Roberts and Ormond 1987; Babcock 1991; Bak and Meesters 1998; Naumann et al. 2009).

In this protocol, two examples are given: one for a branching Porites compressa colony, and one for a massive/mounding Porites lobata colony. There are three parts to estimating colony surface area: 1) photographing coral fragments, 2) image analysis, and 3) calculation of colony surface area using geometric equations.

This protocol was written by Rowan McLachlan and was reviewed by Dr. Andréa Grottoli.

Acknowledgments: I would like to thank Dr. Mia Hoogenboom and Dr. Allison Paley for introducing me to this geometric method at James Cook University, Queensland, Australia.

\section{References:}

- Babcock RC (1991) Comparative demography of three species of scleractinian corals using age- and size-dependent classifications. Ecol Monogr 61:225-244

- Bak RPM, Meesters EH (1998) Coral population structure: the hidden information of 
- Naumann MS, NiggI W, Laforsch C, Glaser C, Wild C (2009) Coral surface area quantification-evaluation of established techniques by comparison with computer tomography. Coral Reefs 28:109-117

- Odum EP, Kuenzler EJ, Blunt MX (1958) Uptake of P32 and primary productivity in marine benthic algae. Limnol Oceanogr 3:340-345

- Roberts CM, Ormond RFG (1987) Habitat complexity and coral-reef fish diversity and abundance on Red-Sea fringing reefs. Mar Ecol Prog Ser 41:1-8

- Szmant-Froelich A (1985) The effect of colony size on the reproductive ability of the Caribbean coral Montastraea annularis (Ellis and Solander). Proc 5th Int Coral Reef Symp 4:295-300

\section{MATERIALS}

\section{Equipment:}

Digital Camera + Memory Card

Tripod

Light Source

Ruler

\section{Software:}

Image J

Microsoft Excel

\section{Photographing corals}

1 Prepare the photograph area. Setup a tripod so that the digital camera is pointing downward, parallel to the workbench as shown in Fig. 1. Place a metric ruler on two blocks of equal height (at least two inches) and make sure a section of the ruler is visible in the digital camera field of view. Arrange a light source so that coral fragments will be evenly lit. If a light source is not available, then the in-built camera flash is also suitable. It is important that all of the coral tissue on the uppermost surface is evenly illuminated (i.e., no dark shadows). Adjust the camera setting such that the ruler is clearly in focus (note: switch to manual focus if using a DSLR camera). If coral samples will be removed directly from experimental tanks (i.e., not frozen), then you may consider placing a waterproof sheet below the tripod, to protect the workbench from saltwater. 


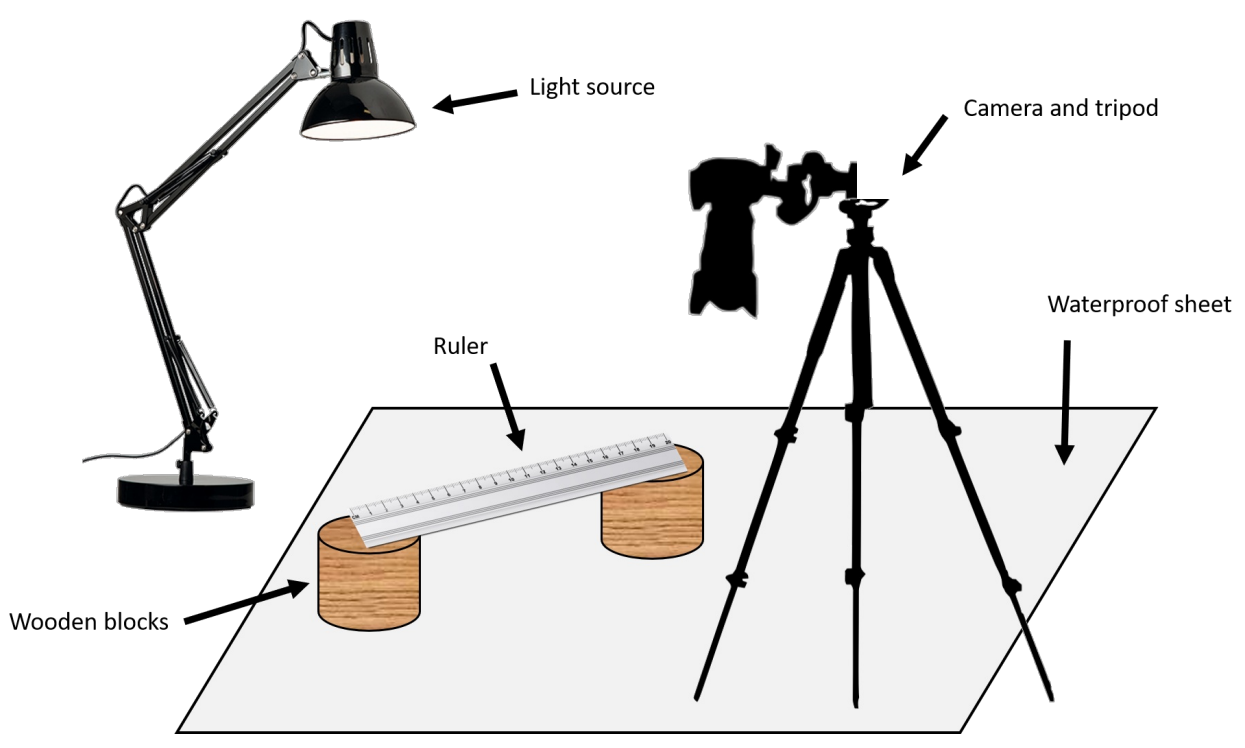

Fig. 1. Set up of photographing area for imaging coral samples.

2 Photograph coral colonies from multiple angles. Remove corals from experimental tanks and proceed to the photographing area. Note: It is essential that the photographs are taken relatively quickly (i.e., $<10$ mins) to prevent prolonged stress to the coral colony due to aerial exposure. Photographs can be taken by holding the coral by hand or placing the fragment on the workbench. Regardless, in order to make accurate measurements, it is vitally important that the uppermost surface of the coral being imaged is at the same height as the ruler (i.e., not above or below the ruler). Take at least six photographs: the four sides taken at right angles to one another (Fig. 2a-d), the top (Fig. 2e), and the base of the colony (Fig. 2f). Try to be as accurate as possible when twisting the coral fragment so that the four side photographs are $90^{\circ}$ (i.e., perpendicular) to one another (Fig. $2 a-d$ ). Likewise, the top and bottom photographs should be $180^{\circ}$ to one another (Fig. 2e, f). 


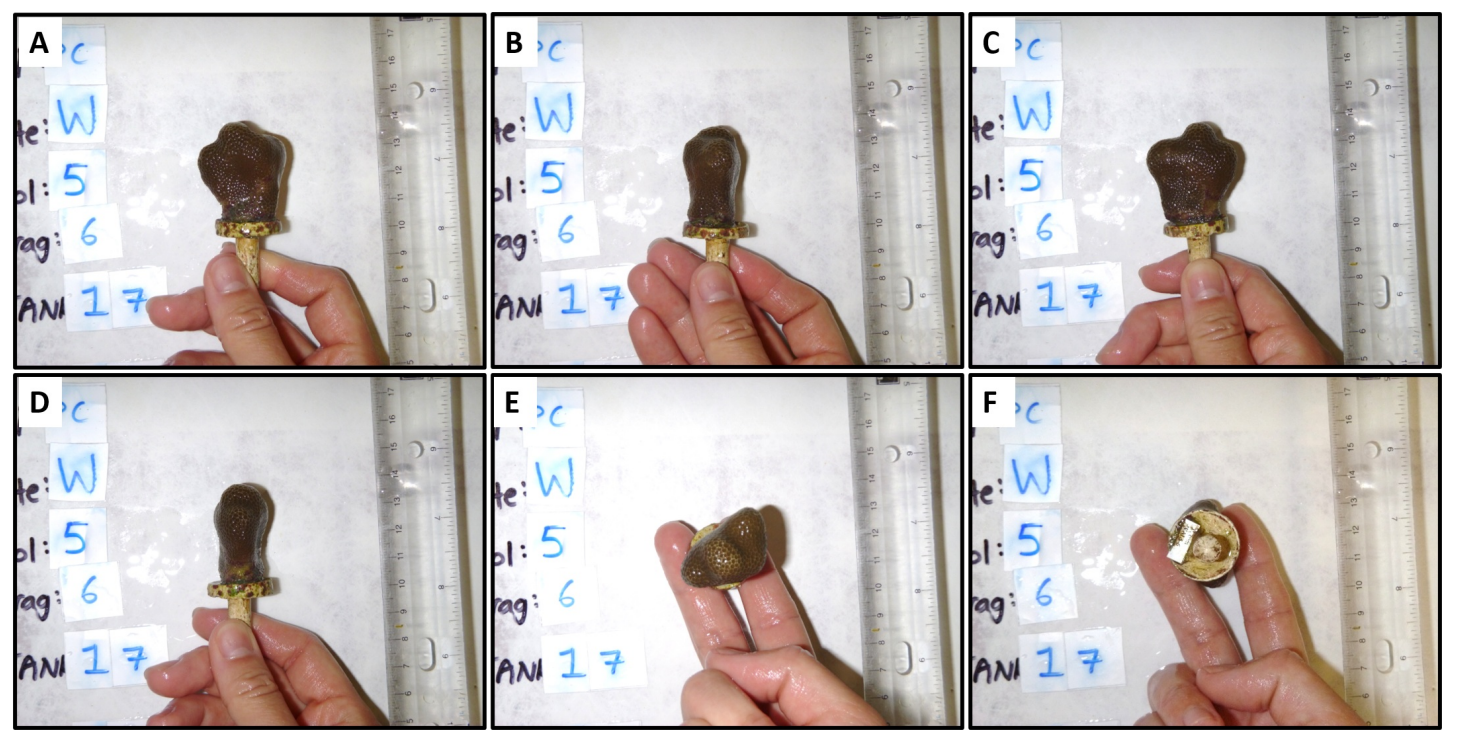

Fig. 2. Photographs of a Hawaiian Porites compressa colony taken from the A) front, B) left, C) back, D) right, E) top, and F) bottom point of views.

When all photographs have been taken, return coral colonies to the experimental tanks.

\section{Image analysis for branching morphology}

3 Overview of a geometric strategy for corals with simple branching morphology

(details in steps 4-10). Several different geometric shapes can be used with this method of estimating the surface area of branching corals including rectangles or cylinders. However, for small fragments of Hawaiian corals species (e.g., Porites compressa and Pocilloproa meandrina), I have found that a truncated rectangular pyramid (Fig. 3A) most accurately approximates the surface area. This method involves measuring widths and heights from two perpendicular images of a coral fragment (Fig. 3B, C), and a 2-dimensional trace of the upper surface of the coral (Fig. 3D). 

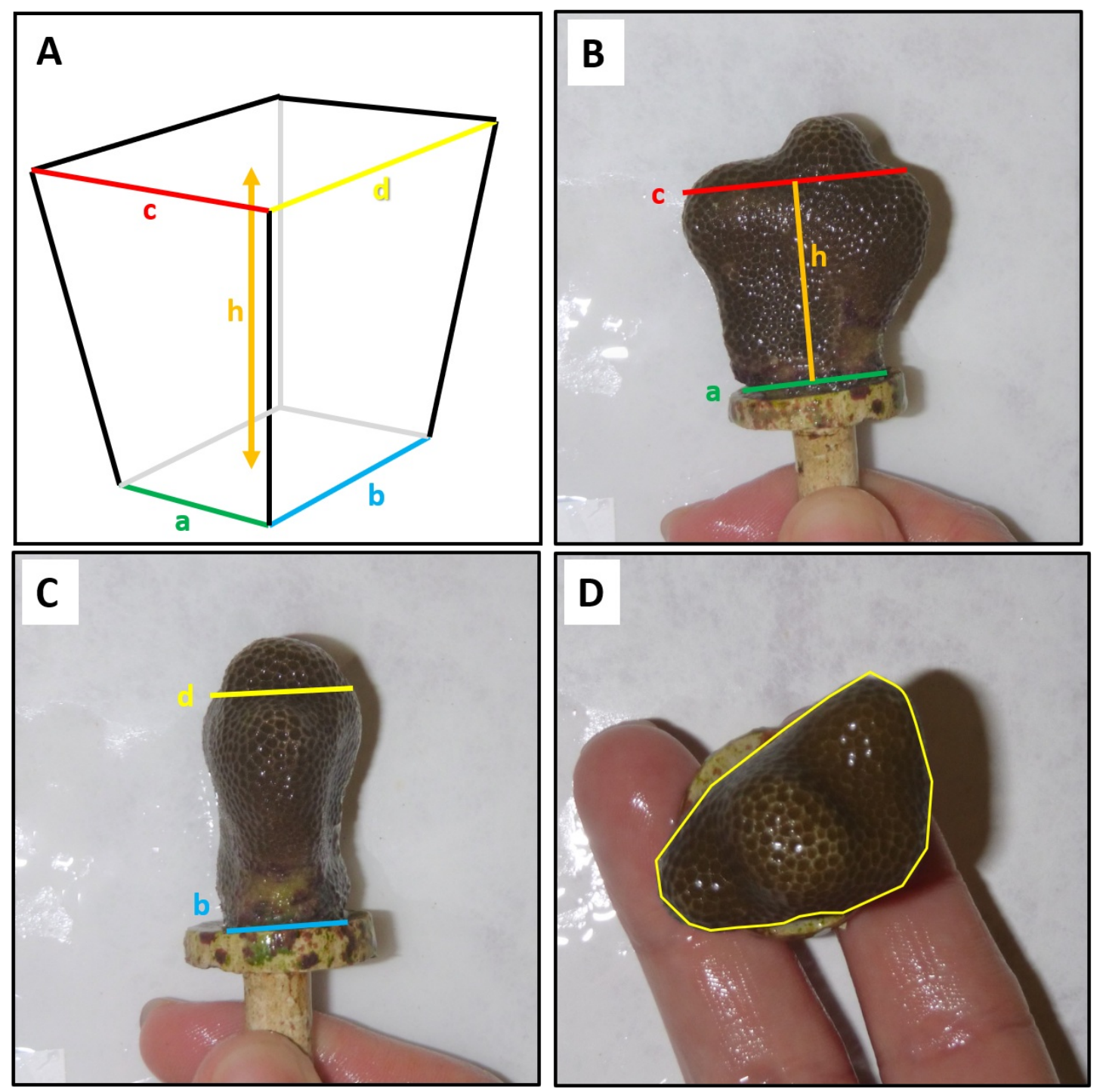

Fig. 3. A) An example of a rectangular pyramidal frustum. B) Digital image of a Porites compressa fragment taken at the front point-of-view. Red, green, and orange lines correspond to the top width, bottom with, and height measurements (respectively) needed for the rectangular pyramidal frustum surface area equation. $\mathbf{C}$ ) Digital image of a Porites compressa fragment taken from the side point-of-view (i.e., perpendicular to the front point-of-view). Yellow and blue lines correspond to the top width and bottom width measurements needed for the rectangular pyramidal frustum surface area equation. D) Digital image of a Porites compressa fragment taken from the top point-of-view. Yellow line polygon trace around the top of the fragment is also known as the dripline or crown area.

4 Download ImageJ. ImageJ can be downloaded for free from the NIH website: https://imagej.nih.gov/ij/download.html. 
5 Import the first photo file. Open the first photo of either the front or the back of the coral (i.e., Fig. $2 \mathrm{~A}$ or Fig. $2 \mathrm{C}$ ) into the Image J software.

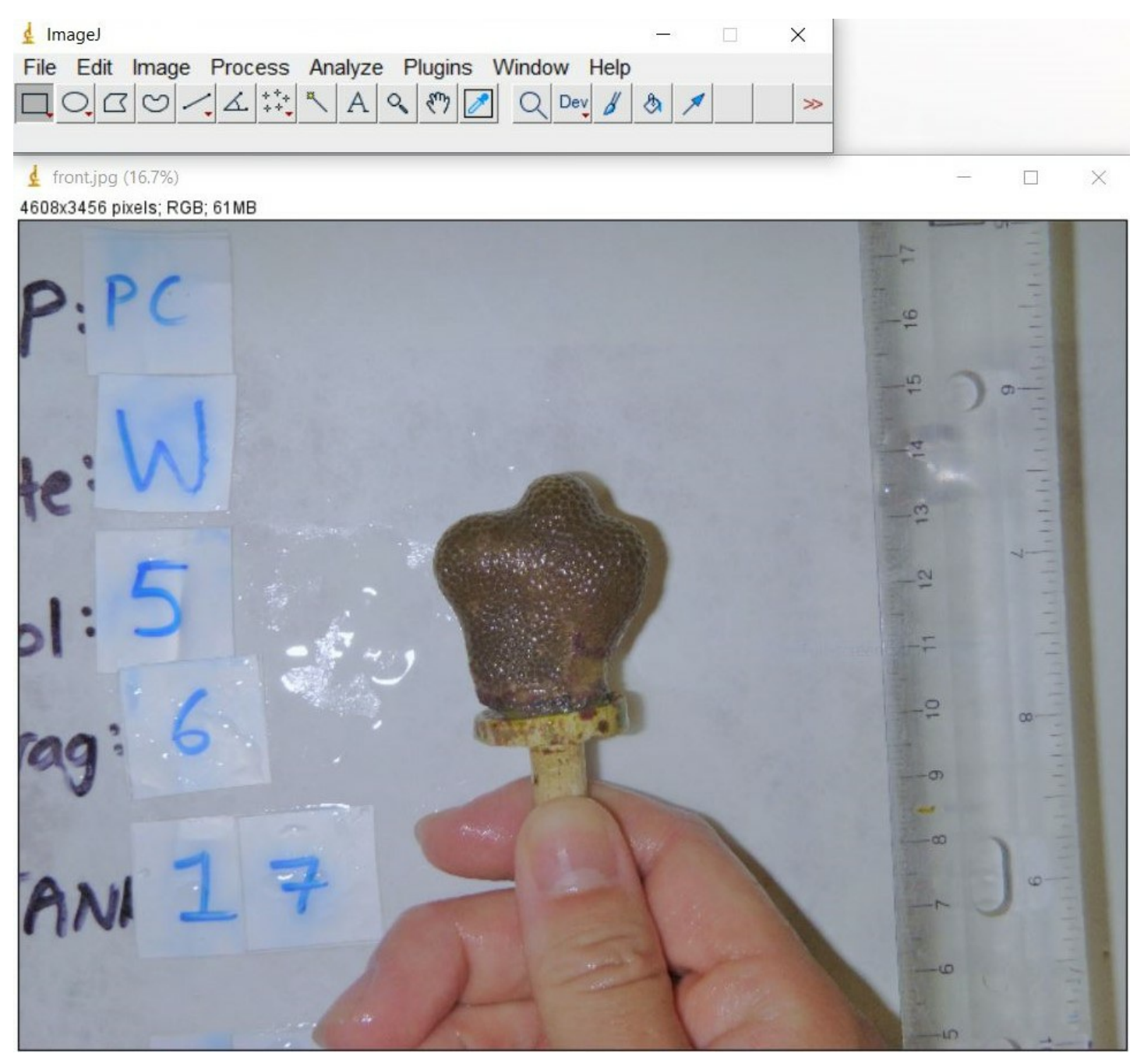

Fig. 4. Digital image of Porites compressa fragment mounted on a ceramic plug photographed next to a ruler, opened in the software ImageJ. Photo taken from the front point-of-view.

6 Set scale. By holding Ctrl + scrolling on your mouse, zoom into a region of the ruler (Fig. 5A). Using the straight-line tool (fifth button from the right in the ImageJ toolbar), carefully highlight a 2 $\mathrm{cm}$ region of the ruler (yellow line, Fig. 5B). Next click on Analyze $>$ Set Scale, after which a dialogue box will appear (Fig. 5C). Set the known distance and unit of length to 2 and $\mathrm{cm}$, respectively (Fig. 5C). 


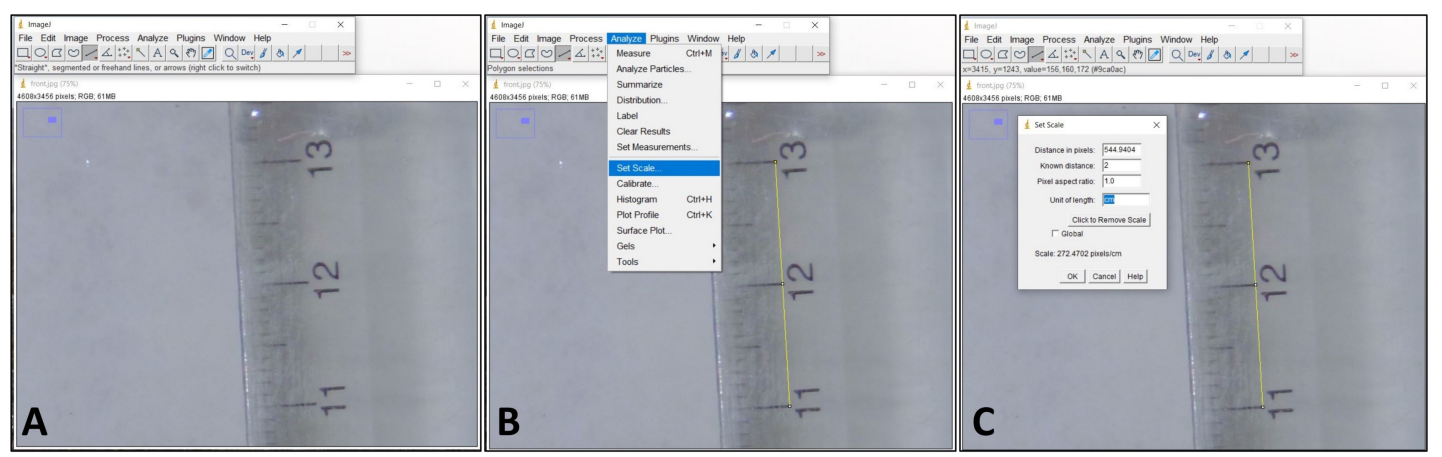

Fig. 5 A) Magnified $\sim 2 \mathrm{~cm}$ region of ruler, B) menu options for setting scale: Analyze > Set Scale, and $\mathbf{C}$ ) dialog box in which to enter the known distance and unit of length.

7 Draw isosceles trapezoid. An isosceles trapezoid has two parallel sides with equal angles from each of the parallel sides (i.e., green and orange angels in Fig. 6A). Using the polygon selection tool (third button from the right in the Image $J$ toolbar), carefully draw an isosceles trapezoid as accurately as possible around the coral fragment (yellow lines Fig. 6B), and then press $C$ trl $+D$ to permanently draw this outline onto the image (black dotted line Fig. $6 \mathrm{C}$ ).

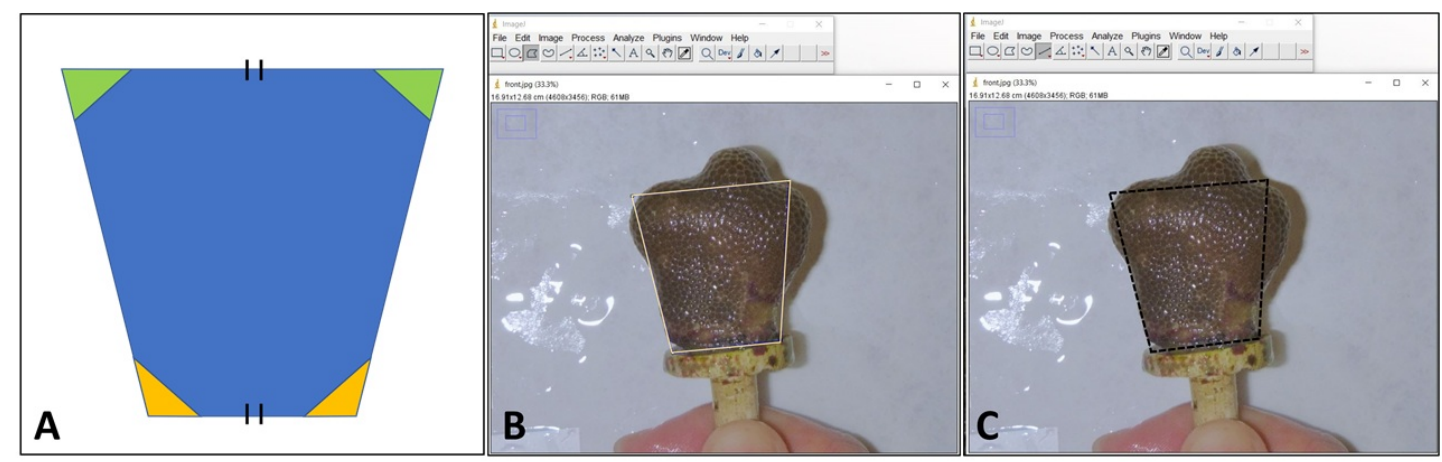

Fig. 6A) An example of an isosceles trapezoid, B) hand-drawn isosceles trapezoid around coral fragment, and C) the black dotted line will be drawn on the photo after pressing Ctrl + D.

8 Measure lengths \& areas. Using the black dotted line guide that you drew in step 7 (Fig. 6C), measure the bottom width (Fig. 7A), top width (Fig. 7B), and height (Fig. 7C) of the coral fragment. Trace each line using the straight-line segment tool, then press $\mathrm{Ctrl}+\mathrm{M}$ to measure the length. $\mathrm{A}$ dialogue box showing the results will appear on the right. If there are any dead tissue patches on 
the coral skeleton, trace around these using the polygon tool and measure (Fig. 7D). Note lengths will be listed under the "Length" column in the results dialogue box, whereas the dead tissue patch area is listed in the "Area" column.

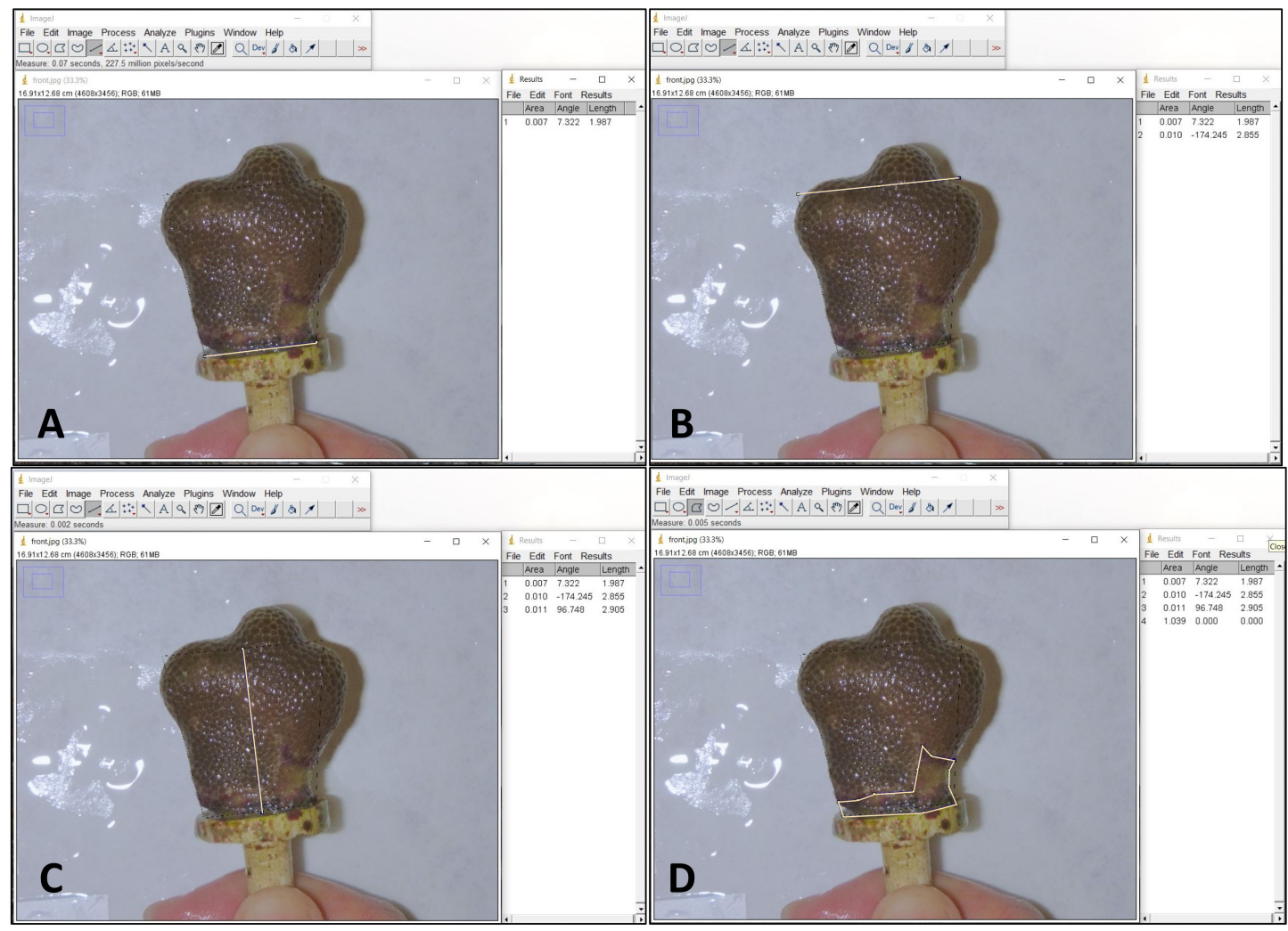

Fig. 7. Line trace of trapezoid A) bottom width, B) top width, C) height, and D) dead tissue area.

9 Repeat for the second photo file. Import the second side photo into ImageJ (Fig. 8A), set the scale (Fig. 8B), draw a new trapezoid (Fig. 8C), and measure the widths, height, and dead tissue area (Fig. $8 D-G$ ) using the methods described in steps 5 through 8 above. 


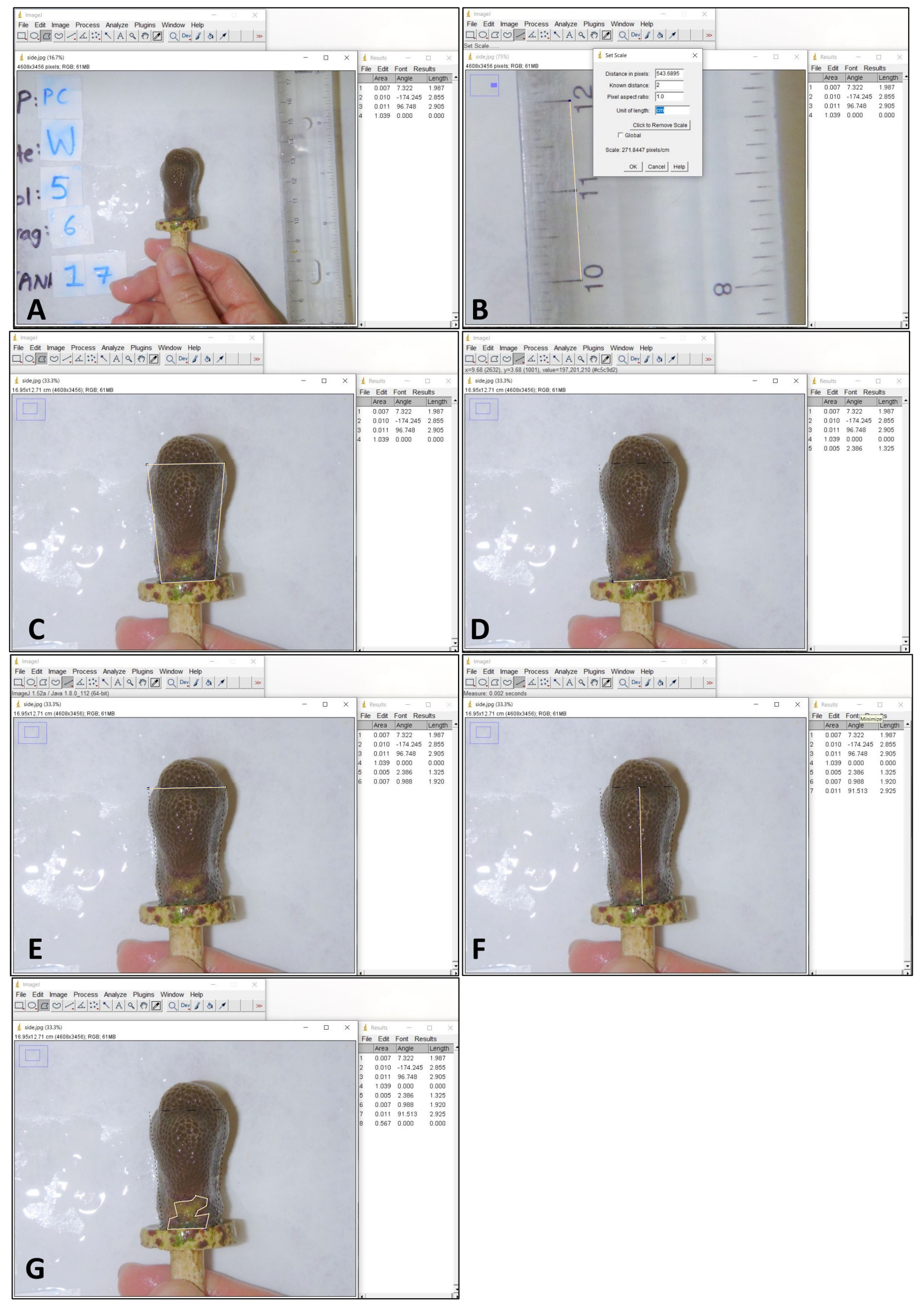

Fig. 8 A) Digital image of Porites compressa fragment taken from the side point-of-view (i.e., perpendicular angle to the front point-of-view). B) Magnified $\sim 2 \mathrm{~cm}$ region of the ruler, C) handdrawn isosceles trapezoid around coral fragment, and line trace of trapezoid $\mathbf{D}$ ) bottom width, E) top width, F) height, and G) dead tissue area. 
10 Measure top photo. Import the top-view photo into ImageJ (Fig. 9A), set the scale (Fig. 9B), and measure the 2-dimensional arial surface area (also known as dripline or crown area) using the polygon tool (Fig. 9C).

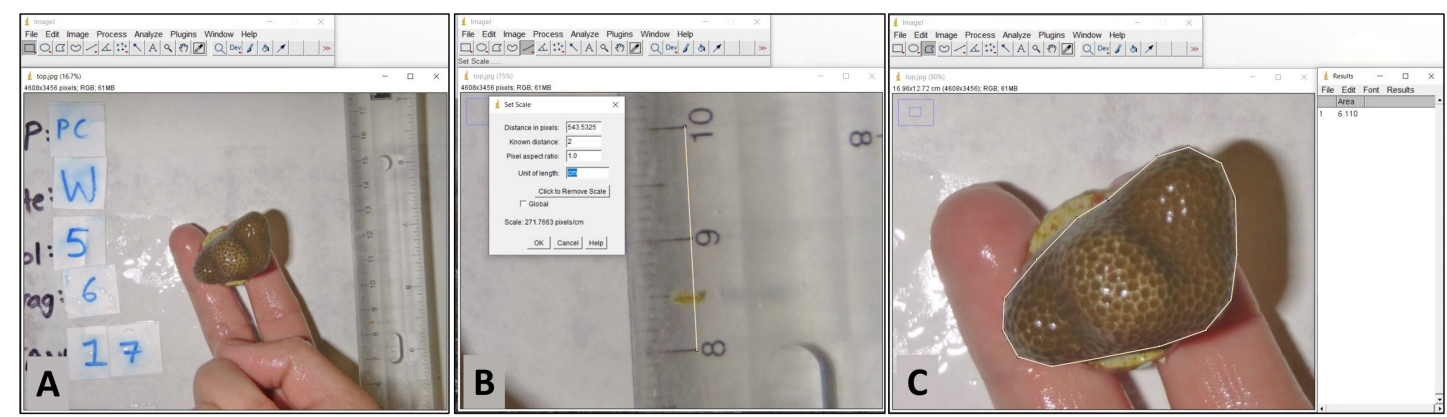

Fig. 9 A) Digital image of Porites compressa fragment taken from the top point-of-view. B) Magnified $\sim 2 \mathrm{~cm}$ region of the ruler. C) Polygon trace (i.e., yellow line) around the top of the fragment (also known as the dripline or crown area).

\section{Image analysis for mounding morphology}

11 Overview of a geometric strategy for corals with simple mounding morphology. Several different geometric shapes can be used in this method of estimating the surface area of mounding corals including circular domes or spheroids. However, for small mounds of Hawaiian corals species (e.g., Porites lobata and Porites evermanni), I have found that half a scalene ellipsoid (Fig. 10A) most accurately approximates the surface area. This method involves measuring widths and heights from two perpendicular images of a coral mound (Fig. 10B, C). 

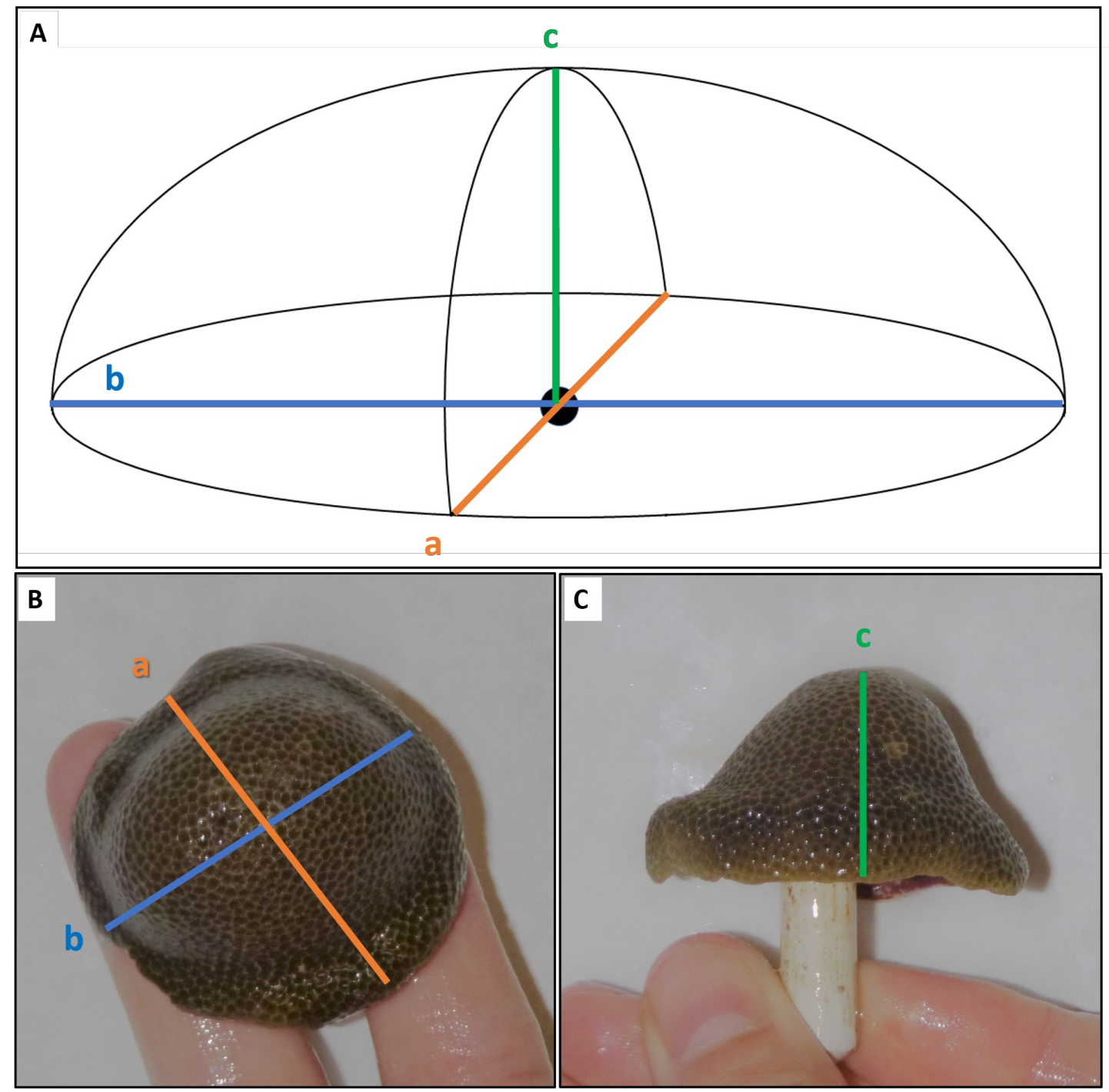

Fig. 10 A) An example of a half a scalene ellipsoid. B) Digital image of Porites lobata mound taken from the top point-of-view. The orange and blue lines correspond to the length (orange line a) and width (blue line b) measurement needed for the half scalene ellipsoid surface area equation. C) Digital image of a Porites lobata mound taken from the side point-of-view (i.e., perpendicular to the top point-of-view). The green line c corresponds to the height measurement needed for the half scalene ellipsoid surface area equation.

12 Import photos, set scale, measure lengths a, b, and $\mathrm{c}$ in Image J using methods described above.

\section{Geometric equations}


The equation for the lateral* surface area of a rectangular pyramidal frustum was found on this website

* Note the lateral surface area does not include the top or bottom surfaces, and instead only the sides. The dripline trace value is used for the upper surface area, and there is no bottom surface area, as the coral colony is attached to the ceramic frag plug. Equations may need to be adapted, depending upon the morphology of your coral samples.

Lateral Surface Area of Rectangular Pyramidal Frustum $\left(\mathrm{cm}^{2}\right)=(a+c) \times \sqrt{ }\left(1 / 4 \times(b-d)^{2}+h^{2}\right)+(b+d)$ $x \sqrt{ }\left(1 / 4 \times(a-c)^{2}+h^{2}\right)$

Calculate the surface area of the branching coral fragment using the $a, b, c, d$, and $h$ values measured, and the equation shown above. Subtract any dead tissue areas, and add on the dripline area for the top of the fragment using the following equation:

Branching Coral SA $\left(\mathrm{cm}^{2}\right)=$ Lateral SA of Rectangular Pyramid + dripline area trace - dead tissue traces

\section{Mounding Morphology - Half Scalene Ellipsoid:}

The equation for the surface area of a scalene ellipsoid* was found on this website.

* Note: this value needs to be divided by two, as the mounding coral represents only half a full scalene ellipsoid.

Surface Area of a Half Scalene Ellipsoid $\left(\mathrm{cm}^{2}\right)=0.5 \times 4 \pi\left[\left(a^{\mathrm{Z}} \times b^{\mathrm{Z}}+a^{\mathrm{Z}} \times c^{\mathrm{Z}}+b^{\mathrm{Z}} \times c^{\mathrm{Z}}\right) / 3\right]^{(1 / \mathrm{Z})}$, where $\mathrm{Z}=1.6075$

Calculate the surface area of the mounding coral fragment using the $a, b$, and $c$ values measured. Subtract any dead tissue areas using the following equation:

Mounding Coral SA $\left(\mathrm{cm}^{2}\right)=$ SA of Half Scalene Ellipsoid - dead tissue traces 